\title{
Schematherapie: Ein Ansatz zur Behandlung narzisstischer Persönlichkeitsstörungen *
}

\section{Schema Therapy: An Approach for Treating Narcissistic Personality Disorder}

E. Dieckmann, W. Behary

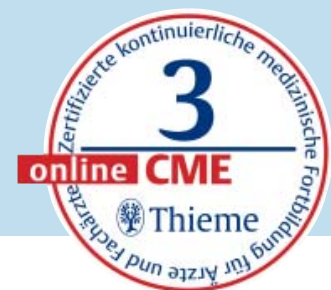

Universitätsklinikum Freiburg, Abteilung für Psychiatrie und Psychotherapie

\section{Zusammenfassung}

$\nabla$

Dieser Artikel soll einen Überblick über die geschichtlichen Hintergründe des Narzissmuskonstruktes sowie die Diagnosekriterien liefern. Es werden verschiedene ätiologische Modelle diskutiert und das schematherapeutische Modell von Jeffrey Young elaboriert. Young entwickelte die Schematherapie (ST) als eine Alternative zur kognitiven Verhaltenstherapie für Menschen mit Persönlichkeitsstörungen. ST unterscheidet sich von den kognitiven Standardtherapien in einigen zentralen Aspekten, wie bspw. der Sicht der Therapiebeziehung als Agens für die Bereitstellung „begrenzter elterlicher Fürsorge“. Außerdem liegt der Fokus des Ansatzes auf der Erfüllung zentraler Grundbedürfnisse des Patienten und der Einbeziehung emotionsaktivierender Techniken, zusätzlich zu kognitiven und behavioralen. Im Artikel werden Youngs Theorie der basalen menschlichen Grundbedürfnisse, frühen maladaptiven Schemata und Schemamodi diskutiert. Aus Sicht der Schematherapie entsteht die narzisstische Persönlichkeitsstörung (NPS) durch Traumatisierung in der Schemadomäne, die die Bindungsbedürfnisse abbildet. Menschen mit NPS tendieren zu starker Verletzlichkeit in Gefolge kränkender Ereignisse, zeigen diese vulnerable Seite jedoch nicht. Stattdessen benutzen sie maladaptive Copingstrategien wie den Modus der Selbstüberhöhung und den Modus des distanzierten Beschützers im Sinne einer Selbstwert stabilisierenden Strategie. Hier präsentieren sie sich als überlegen und arrogant oder von süchtigem oder zwanghaft anmutendem Verhalten geleitet. Diese Konzepte werden anhand von Patientenbeispielen illustriert.

\section{Abstract \\ $\nabla$}

In this article, we review the history of the construct of narcissism and the diagnostic criteria for narcissistic personality disorder. We then discuss some etiological models of narcissism and introduce the model of Jeffrey Young, who developed Schema Therapy (ST) as an alternative to standard cognitive therapy for patients with personality disorders. ST differs from standard cognitive therapies in important respects, including limited reparenting, a focus on the patient's basic needs, and emotional activating techniques in addition to cognitive and behavioral ones. We then discuss Young's theory of basic needs, early maladaptive schemas, and schema modes. According to ST theory, narcissists are traumatized in the schema domain having to do with attachment needs. They are prone to vulnerable emotions in response to narcissistic injuries, although they often do not show these emotions directly. Instead, they use maladaptive coping strategies, resulting in emotional states, known as "schema modes". This includes the Self-Aggrandizer mode and Detached Self-Soother mode, in which a superior, arrogant self-presentation and addictive or compulsive behavior serve a self-regulatory function. These concepts are illustrated by case examples of patients with Narcissistic Personality Disorder.
VNR

2760512015147124917

\section{Bibliografie}

Dol http://dx.doi.org/ 10.1055/s-0035-1553484

Fortschr Neurol Psychiatr 2015; 83: 463-478 @ Georg Thieme Verlag KG Stuttgart · New York . ISSN 0720-4299

\section{Korrespondenzadresse}

\section{Dr. med. Eva Dieckmann}

Praxis für Psychiatrie und Psychotherapie

Stadtstraße 14

79104 Freiburg

Eva.Dieckmann@dieckmannfreiburg.de

\footnotetext{
* Erstveröffentlichung in PSYCH up2date 2010; 4: 53-68
} 


\section{Lernziele}

$\nabla$

- Einblick in unterschiedliche Konzepte zur Narzissmusbehandlung erhalten

- Überblick über die wichtigsten ätiologischen Konzepte der Schematherapie für die narzisstische Persönlichkeitsstörung darstellen

- Heranführung an die zentralen Therapiestrategien der Schematherapie für die narzisstische Persönlichkeitsstörung

\section{Einleitung}

Gängige Therapiemethoden gelten für Patienten mit Persönlichkeitsstörungen gemeinhin als wenig effektiv, woraus im Umgang mit dieser Patientengruppe nicht selten ein latenter therapeutischer Nihilismus resultiert. Fiedler [1] warnt explizit vor dem damit einhergehenden Problem der „selbsterfüllenden Prophezeiung“ des Therapieversagens. Insbesondere trifft dies für die narzisstische Persönlichkeitsstörung (NPS) zu. Lorna Smith Benjamin, ausgewiesene Expertin auf diesem Gebiet, hat sich im Rahmen einer erfrischend formulierten Selbstöffnung folgendermaßen geäußert [2]: „Die feine Kunst, beim narzisstischen Persönlichkeitsgestörten den Rand des Bewusstseins zu erweitern, ohne die therapeutische Beziehung zu zerstören, ist schwer zu beschreiben. Ich habe viele von Narzissmus Betroffene in die Flucht gejagt und beginne erst in jüngster Zeit, mich einer Beherrschung des Problems anzunähern."

Der charakteristische interpersonelle Stil dieser Patientengruppe stellt hohe Anforderungen an die therapeutische Beziehungsgestaltung, auch wenn ihr Leidensdruck unübersehbar ist.

Es dominiert eine typische Anspruchshaltung, gepaart mit einem oft eklatanten Mangel an Mitgefühl, rascher Kränkbarkeit und Neigung zu Schuldzuweisungen. Darüber hinaus besteht eine Tendenz zu bedrohlichem und aggressivem Verhalten und einem erhöhten Delinquenzrisiko [3].

Diese Konstellation provoziert nicht selten ungünstige Reaktionen bei Therapeuten, die sich schädlich auf die Behandlung auswirken können. Erschwerend kommt dabei hinzu, dass die Patientengruppe häufig eine untrügliche Intuition für die Verletzlichkeiten ihres Gegenübers aufbringt. Zitat eines unter NPS leidenden Patienten:

„Wenn es um die Schwächen und Empfindlichkeiten meines Gegenübers geht, bin ich wie ein Trüffelschwein. Ich finde sie alle heraus. Und kann dadurch so furchtbar verletzend werden, dass ich schon viele Menschen schockiert habe."

So wird des Öfteren übersehen, dass die Patienten hinter ihrer als arrogant wahrgenommenen Fassade meist ein hohes Maß an Verunsicherung ver- bergen. Der Therapeut sollte dementsprechend in der Lage sein, im Sinne einer wertschätzenden Irritation Mitgefühl und Respekt mit Grenzsetzung und Konfrontation flexibel kombinieren zu können. Dies kommt für gewöhnlich einem komplexen Balanceakt gleich und stellt an den Behandler hohe Ansprüche in Bezug auf Expertise, Reaktionsvermögen, Authentizität und das Bewusstsein eigener Überempfindlichkeiten.

Man sollte auch bedenken, dass die Inzidenz sowohl narzisstischer Persönlichkeitszüge wie auch der NPS in den vergangenen Jahren zugenommen hat $[4,5]$. Vor dem Hintergrund der zunehmenden Bedeutung dieses Störungsbildes ist es bemerkenswert, dass systematische empirische Studien zur seiner Behandlung noch nicht existieren. Dies ist wahrscheinlich nicht weiter verwunderlich. Forschung mit Patienten zu betreiben, deren Hauptcharakteristikum darin besteht, sich nicht an die Regeln halten zu wollen, ist sicherlich kein leichtes Vorhaben. Den meisten Überlegungen zur Ätiologie und Behandlung dieses Phänomens liegen klinische Beobachtungen, Fallbeschreibungen und Fallanalysen zugrunde. Es existiert keine randomisiert kontrollierte Therapiestudie, die ausschließlich narzisstische Patienten einschloss, meist sind die Patientenstichproben sehr gemischt, und Persönlichkeitsstörungen werden eher im Sinne einer komplizierenden Bedingung betrachtet. Hier besteht ein dringender Handlungsbedarf.

Im vorliegenden Artikel werden die ätiologischen Modelle unterschiedlicher Therapieschulen beschrieben und zeitgemäße Behandlungsansätze, insbesondere solche, die Einfluss auf den schematherapeutischen Ansatz zur Therapie der NPS haben, erwähnt. Der Fokus liegt dabei auf dem Erklärungsansatz der Störung und den daraus abgeleiteten praktischen Interventionsvorschlägen der Schematherapie nach Jeffrey Young.

\section{Historischer Hintergrund des Narzissmusbegriffes \\ $\nabla$}

Der Begriff „Narzissmus“ geht auf den römischen Dichter Ovid zurück. In seinen „Metamorphosen“, die sich dem Phänomen der Verwandlung in unterschiedlichen Facetten widmen, berichtet er über den potenziell tödlichen Verlauf dieser Störung. Das über 2000 Jahre alte Werk enthält in seiner verdichteten Form zahlreiche Elemente, die auch für die kontemporären Ausgestaltungen narzisstischer Thematiken Gültigkeit haben. Echo, eine Bergnymphe, offenbart Narziss, einem wunderschönen Jüngling, ihre Zuneigung: Aber er flieht und entreißet im Fliehn der Umschlingung die Hände: „Eher“, so ruft er, „den Tod, als dass du mir nahtest in Liebe! “

Echo ist von seiner barschen Abfuhr so verletzt, dass sie dahinsiecht, bis nur noch ihre Stimme und ihre Knochen von ihr übrig bleiben. Narziss 
produziert durch seine Gefühlskälte weitere Opfer, wovon eines ihn verflucht:

„So mag lieben er selbst, so nie das Geliebte besitzen!“

Dieser Verwünschung schließt sich Nemesis an, in der griechischen Mythologie die Göttin des gerechten Zorns, zu deren Aufgaben die Bestrafung „herzlos Liebender“ gehört. Narziss wird dazu verurteilt, sich in sein eigenes Spiegelbild im Wasser zu verlieben. Gebannt sucht er die Annäherung an sein Ebenbild, doch es verschwimmt, wenn er sich nähert und die Wasseroberfläche berührt. Verzweifelt konstatiert er:

„Was kein Liebender wünscht, ich wünsche mir fern, was ich liebe."

Schließlich stirbt er, unfähig, sich von seinem Ebenbild loszureißen. An der Stelle seines Todes wächst eine Blume, die seinen Namen trägt.

\section{Diagnostische Kriterien \\ $\nabla$}

Es ist bezeichnend, wie viele Überschneidungen es in der historischen Annäherung an die Störung und den aktuellen DSM-IV-Diagnosekriterien gibt, und wo die Unterschiede liegen.

In die gegenwärtige Interpretation des Narzissmus haben Einzug gehalten die ausgeprägte Ich-Bezogenheit, angereichert um die daraus erwachsenden Themen wie Neid, übertriebenes Selbstwertgefühl, Sucht nach Bewunderung, Erfolg und Macht. Auch der beschriebene Mangel an Empathie findet Erwähnung. Das Hauptproblem des Namensgebers der Störung fehlt: das Unvermögen, authentische und reziproke Beziehungen einzugehen und das ambivalent empfundene Bedürfnis nach Distanz zum Gegenüber.

Der Auswahl dieser Diagnosekriterien wird angelastet, dass sie zu sehr auf die Kompensationsstrategien abhebt, die die Patienten anwenden, um ihr brüchiges Selbstwertgefühl zu kompensieren. Damit trage sie zu einer negativen Sichtweise einer Patientenpopulation bei, die von vorneherein bei vielen Therapeuten Unsicherheit oder Ablehnung hervorrufe und verhindere ein ganzheitlicheres Verständnis der Problematik [6]. So wird oft übersehen, dass sich nicht selten hinter der in verschiedener Aufmachung präsentierten abschreckend wirkenden Seite der Patienten - sei sie nun ansprüchlich, abwertend, bedrohlich oder von zur Schau getragener Großartigkeit geprägt - Einsamkeit, innere Leere und Leid befinden. Dieser Leidensdruck findet im DSM-IV jedoch keinen Niederschlag. Auch bleibt anzumerken, dass narzisstische Störungen sich oft sehr viel subtiler zeigen. Cooper und Ronningstam beschreiben als Subtyp den „covert narcissist“, der zurückgezogen, scheu und inhibiert wirkt und seine Größenideen nur in der Phantasie auslebt [7].

\section{Kontemporäre ätiologische und therapeutische Konzepte $\nabla$}

\section{Soziale Lerntheorie nach Millon}

Millons soziale Lerntheorie der NPS stützt sich in erster Linie auf elterliche Überbewertung als ätiologischen Faktor [8]. Die späteren Patienten hätten gelernt, ausnahmslos liebenswert und perfekt zu sein [9]. Dementsprechend finden sich auch hier vor allem Beschreibungen kompensatorischer Strategien, auf die Beziehungsstörung wird nur kursorisch eingegangen [10].

\section{Interpersonelle Ansätze}

Timothy Leary formulierte 1957 in seinem Buch „Interpersonal diagnosis of personality“ einen interpersonellen Ansatz der Problematik von Persönlichkeitsstörungen [11]. Eine Reihe von interpersonellen Theoretikern folgte seinen Ausführungen, wobei die Arbeiten von Lorna Smith Benjamin hervorstechen. In ihrem Buch „Die interpersonelle Diagnose und Behandlung von Persönlichkeitsstörungen“ leitet sie eine komplexe Analyse narzisstischer Charaktereigenschaften her. Als hauptursächlich für die Entwicklung der NPS sieht sie einen Erziehungsstil, der selbstlose Vergötterung mit bewundernder Hingabe kombiniert bei gleichzeitigem Verzicht auf elterliche Selbstoffenbarung. Aus ersterem folge die Sucht des späteren Patienten nach unermüdlicher Bewunderung, aus letzterem resultiere das Unvermögen des Narzissten, anderen Menschen eigene Bedürfnisse und Wünsche zu gewähren. Gleichzeitig habe jedoch für den späteren Patienten die konstante Gefahr bestanden, die Gunst der Eltern zu verlieren, sobald er sich durchschnittlich verhalten hätte. Jede Andeutung von Unvollkommenheit habe gravierende Konsequenzen gehabt.

\section{Therapieziel}

Zum Erreichen des primären Therapieziels, nämlich einer Einsicht des Patienten in dysfunktionale inter-personelle Muster, sei von therapeutischer Seite sorgsam kanalisierte Empathie vorrangig.

Diagnosekriterien des DSM-IV
Die NPS ist gekennzeichnet durch ein tief greifen-
des Muster von Großartigkeit in Phantasie oder
Verhalten, Bedürfnis nach Bewunderung und
Mangel an Empathie.
Der Beginn liegt im frühen Erwachsenenalter und
zeigt sich in verschiedenen Situationen. Mindes-
tens 5 der folgenden Kriterien müssen erfüllt
sein: Der Patient
1. hat ein grandioses Gefühl der eigenen Wich-
tigkeit, übertreibt etwa die eigenen Leistungen
und Talente; erwartet ohne entsprechende Leis-
tungen als überlegen anerkannt zu werden.
2. ist stark eingenommen von Phantasien gren-
zenlosen Erfolgs, Macht, Glanz, Schönheit oder
idealer Liebe.

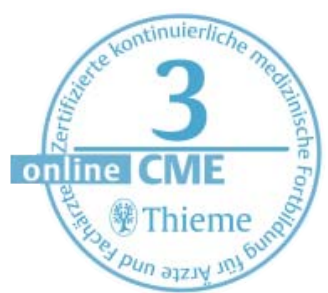


3. glaubt von sich, „,besonders“ und einzigartig zu sein und nur von anderen besonderen Personen oder Institutionen verstanden zu werden oder nur mit diesen verkehren zu können.

4. verlangt nach übermäßiger Bewunderung.

5. legt ein Anspruchsdenken an den Tag, erwartet eine bevorzugte Behandlung oder automatisches Eingehen auf die eigenen Erwartungen.

6 . ist in zwischenmenschlichen Beziehungen ausbeuterisch, zieht Nutzen aus anderen, um eigene Ziele zu erreichen.

7. zeigt einen Mangel an Empathie: ist nicht fähig, die Gefühle oder Bedürfnisse anderer zu erkennen oder sich mit ihnen zu identifizieren.

8. ist häufig neidisch auf andere oder glaubt, andere seien neidisch auf ihn.

9. zeigt arrogante, überhebliche Verhaltensweisen oder Handlungen.

Es muss vermieden werden, dass das Mitgefühl des Therapeuten narzisstische Muster verstärkt, sondern Einsicht begünstigt und Veränderungspotenzial schafft.

Zusätzlich empfiehlt Smith Benjamin, die Zusammenarbeit durch vereinzelt eingestreute Hinweise des Therapeuten auf seine eigene Fehlbarkeit mit gleichzeitiger Äußerung diesbezüglicher Selbstakzeptanz zu erleichtern.

Als weitere Therapieziele sieht sie:

- durch in Unterstützung eingebettete Konfrontationen das Erkennen wiederkehrender Muster zu ermöglichen,

- maladaptive Muster zu blockieren, indem großzügige Unterstützung gewährt wird, wenn der Betroffene sich aktiv bemüht, seine Probleme anzugehen,

- den Willen des Patienten, diese aufzugeben, zu stärken.

- neues Lernen fördern: z. B. durch den Einsatz von Rollenspielen, in denen der Patient die Rolle eines Gegenübers, das Ziel seiner Entwertungen, Angriffe oder Beschuldigungen wurde, spielen soll, um Einsicht in die Folgen seines Verhaltens zu erleichtern.

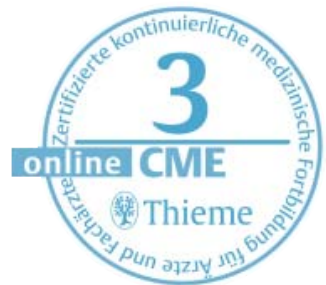

Narzissmuskriterien nach Millon

- Personen mit NPS haben gelernt, dass sie sich mehr auf sich als auf andere verlassen.

- Sie streben danach, besser, schöner, bedeutender und reicher zu sein.

- Sie verhalten sich in sozialen Situationen nicht reziprok, sie erwarten Bewunderung und Hilfe von anderen im Erreichen ihrer Ziele, sehen dies als selbstverständlich an, es steht ihnen zu, sie müssen nichts dazu beitragen oder sich revanchieren.
Sie können sogar annehmen, dass es anderen zur Ehre gereicht, eine Beziehung zu ihnen haben oder ihnen dienen zu dürfen.

- Die Selbstverständlichkeit, mit der sie erwarten, dass man ihre Bedürfnisse befriedigt, macht häufig Eindruck und führt dazu, dass sich Menschen initial reziprok verhalten.

- Sie suchen sich auch Partner aus, die bereit sind, sich für sie einzusetzen, z. B. Partner mit einer abhängigen Persönlichkeitsstörung.

- Da sie immer wieder auf Personen stoßen, die sie unterstützen, sind Personen mit NPS nicht gezwungen, ihr ausbeuterisches Verhalten zu verändern.

- Sie haben mitunter ausufernde Phantasien über eigenen Erfolg, die sich nur schwach an der Realität orientieren.

- Sie sind meist so sehr davon überzeugt, dass sie besondere Personen sind und ihnen deshalb Sonderrechte eingeräumt werden müssen, dass sie diese Annahme nicht infrage stellen. Dadurch reagieren sie irritiert bis ärgerlich auf Personen, die diese Ansicht hinterfragen.

- Sie haben Mechanismen, sich selbst zu bekräftigen: sich selbst zu sagen, wie einzigartig sie sind, Misserfolge umzuattribuieren etc.

- Sie neigen stark dazu, ihre Erinnerungen entsprechend ihren Annahmen zu verzerren: wenn sie von einem Partner im Stich gelassen wurden, dann „erinnern“ sie sich daran, diese verlassen zu haben.

- Wenn sie Schwierigkeiten haben (scheitern, kritisiert werden, nicht erfolgreich sein) wenden sie defensive Mechanismen an. Sie rationalisieren, verwenden Alibis.

- Wenn diese defensiven Strategien scheitern, fühlen sie sich zurückgewiesen, beschämt, depressiv, leer. Sie haben nicht gelernt, mit Misserfolgen konstruktiv umzugehen.

- Sie haben auch nicht gelernt, bei Frustration Trost und Zuwendung bei anderen zu suchen.

- Wenn alle Strategien versagt haben, ziehen sie sich in Größenphantasien zurück, die nichts mit der Realität zu tun haben.

- Sie erleben wenige Konflikte. Sie sind davon überzeugt, dass alles für sie gut läuft.

- Sie reflektieren wenig darüber, was andere Menschen denken.

- Dadurch sind ihre defensiven Strategien leicht durchschaubar.

Fallbeispiel/Praktisches Vorgehen Interpersonelle Ansätze

Ein Patient mit NPS fällt aus allen Wolken, als sich seine Frau in der Paartherapie über ihn beklagt. Die einfühlsame Aussage wie „Sie haben sich so bemüht, dass alles gut läuft, und nun kommt Ihre Frau mit Klagen daher" beinhaltet die potenzielle Gefahr, dass der Patient sich vollständig 
verstanden fühlt und auf seine Strategien der Externalisierung und Beschuldigung zurückgreift. Optimaler wäre folgende Alternative: „Sie haben sich angestrengt, dass alles gut läuft und nun fühlen Sie sich einfach am Boden zerstört, weil Sie hören, dass es nicht so perfekt läuft, wie Sie dachten." Dies wird als herzliche Zusammenfassung der Muster des Patienten mit der sanften Konfrontation, sich dieser zunehmend bewusst zu werden, gewertet.

Dem Patienten wird vermittelt, dass unrealistische Ziele und maladaptive Verhaltensweisen zugunsten fruchtbarerer kognitiver und interaktiver Gewohnheiten aufgegeben werden müssen.

\section{Das Modell der doppelten Handlungsregulation}

Rainer Sachse geht in seinem störungstheoretischen Modell davon aus, dass Persönlichkeitsstörungen als Interaktionsstörungen begriffen werden müssen und die dysfunktionale Beziehungsgestaltung die zentrale Problematik darstellt [12]. Er benennt die Motivebene als die am höchsten in der Hierarchie menschlicher Bedürfnisse angesiedelte Regulationsinstanz. Demnach ist bei der NPS das zentrale Beziehungsmotiv „Anerkennung, Liebe und Zuwendung“ nicht erfüllt worden durch Mangel an Bestätigung oder durch beständiges negatives Feedback. Des Weiteren frustriert wurde der Wunsch nach verlässlichen und solidarischen Beziehungen. Für die Ebene der Grundannahmen resultieren daraus nach Sachse negative Selbstbeschreibungen.

Übertrieben negative Selbstschemata sind etwa:

> „Ich bin nicht okay.“

> "Ich bin ein Versager.“

- „In Beziehungen kann man abgewertet werden." etc.

Es entwickelt sich ein intensives Spannungsfeld zwischen dem hoch in der Motivhierarchie aktivierten frustrierten Grundbedürfnis und den negativen Selbstannahmen.

Als Kompensationsversuch bedient sich die Person laut Sachse der Spielebene: „Die Person kann in ihrem Bezugssystem die Erfahrung machen, dass sie zwar als Person nicht wichtig genommen wird, dass sie jedoch für bestimmte Arten von Verhaltensweisen durchaus Aufmerksamkeit erhalten kann." Für Personen mit NPS besteht die Lösung des Dilemmas in dem Bemühen, durch Leistung Anerkennung zu verdienen. Jedoch wird so die Motivebene nicht gestillt. Überdies wird dadurch ein doppeltes Selbstkonzept mit diametral entgegengesetzten Annahmen und Stimmungszuständen entwickelt. Je nachdem, ob aktuell das biografisch begründete, in einem affektiven Repräsentationscode ge- speicherte negative Selbstschema dominiert oder durch günstiges Feedback das (häufig übertrieben) positive Selbstschema bedient wurde, herrschen extrem negative Kognitionen und Verstimmungszustände vor oder es besteht eine überzogen positive Selbsteinschätzung bei euphorisierter Affektlage.

Übertrieben positive Selbstschemata sind etwa:

- „Ich bin der Kompetenteste.“ statt „Ich bin kompetent.“

• „Ich bin der Tollste.“ statt „Ich bin akzeptabel.“

\section{Therapieziel}

Als therapeutische Ziele formuliert Sachse, die Kommunikation zwischen diesen beiden Schemata zu bewerkstelligen. Auf der Grundlage einer vertrauensvollen Therapeut/ Patientbeziehung soll der Patient darüber hinaus lernen, dass er „ein Teil des Problems“ ist und dass sein Verhalten Kosten verursacht. Weiterhin soll ein $\mathrm{Zu}-$ gang zu seinen zentralen Beziehungsmotiven ermöglicht und das Agieren auf der „Spielebene“ vermindert werden.

\section{Kognitive Therapie}

Nach Beck und Freeman bestehen bei der NPS eine Reihe verzerrter Annahmen über die eigene Person, die Umwelt und die Zukunft, die zwingend und übermäßig aktiv sind [13]. Die narzisstische Person hält sich für einzigartig und außergewöhnlich. Generiert wird diese Überzeugung durch eine elterliche Haltung von Nachsicht und Begünstigungen sowie dem Vorenthalten negativer Rückmeldungen, was die NPS zu einem Feedbackproblem werden lässt. Die Rolle der postulierten Versorgungsdeprivation in der Entwicklung dieser Störung kommentieren die Autoren aufgrund mangelhafter Studienlage eher kritisch: ,Jedoch scheint die psychoanalytische Theorie der narzisstischen Ätiologie ihre Grenzen zu haben aufgrund der übermäßigen Betonung angenommener Unzulänglichkeiten in der emotionalen Versorgung durch die Mutter." Gleichwohl beschreiben sie, dass frühkindliche Opfererfahrungen oder Ausgrenzung ein übersteigert negatives Selbstbild induzieren können, das sozusagen spiegelbildlich zu einem zu positiven Selbsteindruck eine Haltung von Besonderheit unter negativen Vorzeichen abbildet.

\section{Therapieziel}

Therapieziele sind in Einklang mit allen anderen vorgestellten Ansätzen die Notwendigkeit der initialen Entwicklung einer Beziehung, die sich auf Zusammenarbeit stützt. Des Weiteren soll das kognitive Behandlungsmodell erlernt werden. Der Abbau der spezifischen Beschwerden wird als kurzfristiges Ziel benannt, im Verlauf wäre eine Änderung des grandiosen Selbstbildes, ein adaptiver Umgang mit Rückmeldungen anderer, die Förderung des Bewusstseins für die Gefühle der Umwelt sowie die Aufgabe ausbeuterischen Verhaltens zu nennen. Kognitive Techniken zielen

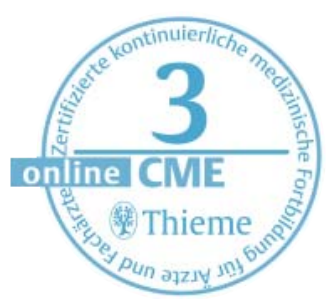




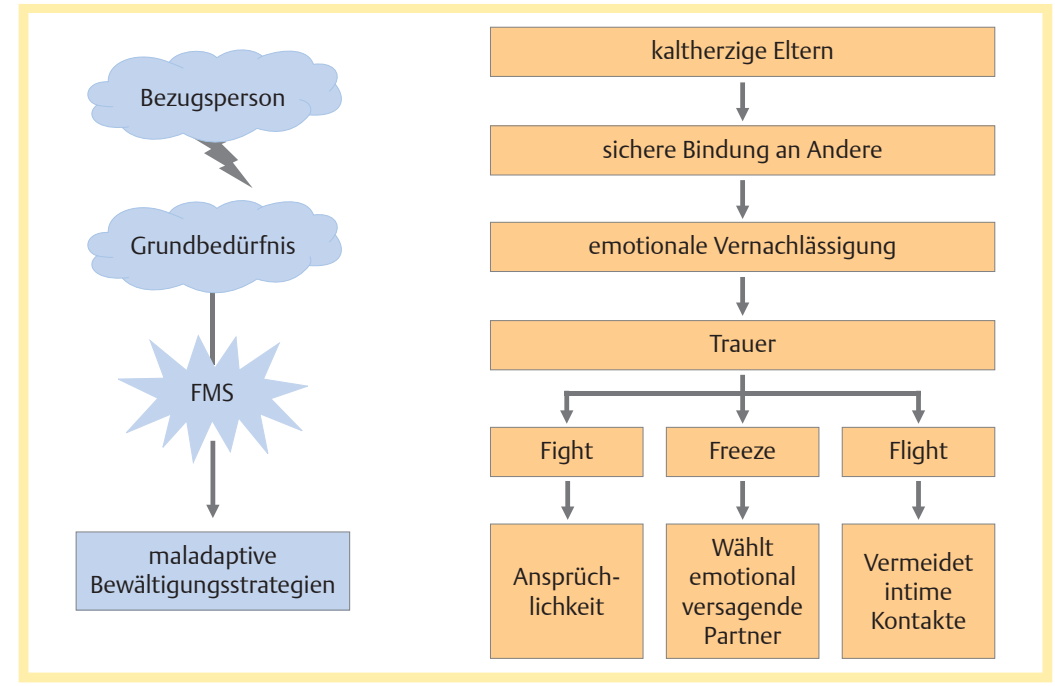

Abb. 1 Bewältigungsstrategien.

auf die Modifikation des ausgeprägten dicho-

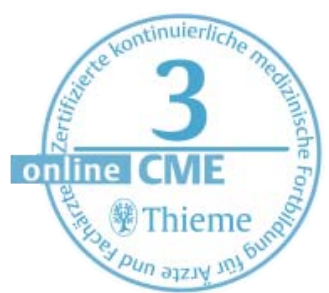
tomen Denkens der Patienten ab sowie auf die Entwicklung alternativer Annahmen und Bilder (z. B. die Vorstellung zu entwickeln, Spaß am Singen im Gemeindechor zu haben statt sich mit Phantasien erfolgreicher Auftritte vor großem Publikum zu tragen). Diese sollten von den genuinen Interessen des Patienten geprägt sein und nicht vom Wunsch nach externem Lob und Aufmerksamkeit.

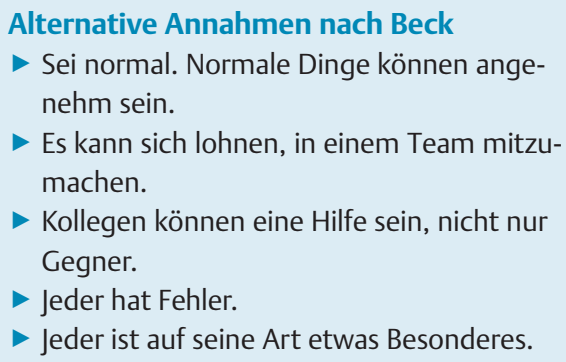

Alternative Annahmen nach Beck

- Sei normal. Normale Dinge können angenehm sein.

- Es kann sich lohnen, in einem Team mitzumachen.

- Kollegen können eine Hilfe sein, nicht nur Gegner.

- Jeder hat Fehler.

- Jeder ist auf seine Art etwas Besonderes.

Bezüglich der Überempfindlichkeit für die Einschätzung anderer empfehlen die Autoren eine Hierarchisierung von Situationen, die mit Rückmeldung und Kritik einhergehen mit konsekutiver systematischer Desensibilisierung durch gestufte Exposition. Rollenspiele, in denen der Patient die Position seines Gegenübers einnimmt, sollen dessen Empathiefähigkeit verbessern.

\section{Schematherapie als integrativer Ansatz}

Jeffrey Young entwickelte die Schematherapie aus der kognitiven Verhaltenstherapie heraus, nachdem er den Schwerpunkt seiner Arbeit auf die Therapie von Menschen mit Persönlichkeitsstörungen verlagerte und hierbei an die Grenzen des traditionellen Ansatzes stieß [14].
Die Schematherapie wurde aus der kognitiven Verhaltenstherapie heraus für Persönlichkeitsstörungen konzeptualisiert.

- Die Schematherapie kombiniert kognitive, verhaltenstherapeutische, emotionsfokussierte und gestalttherapeutische Techniken.

- Dem Stellenwert entwicklungsbedingter traumatischer Erfahrungen und deren Auswirkungen auf aktuelles Denken, Fühlen und Handeln durch Bildung eines autobiografischen Gedächtnisses wird eine hohe Bedeutung beigemessen.

- Die Rolle emotiver Techniken zur Aktivierung von Schemata ist herausragend.

- Starke Gewichtung erfährt die therapeutische Beziehung als wichtigster Motor der Veränderung. Der Ansatz ist bedürfnisbasiert. Da Patienten mit Persönlichkeitsstörungen während ihres Aufwachsens meist mit dysfunktional agierenden Bezugspersonen aufwuchsen, wurden grundlegende Beziehungsmotive frustriert. Die Therapiebeziehung soll zum Antidot für diese Erlebnisse werden und den Patienten mit korrektiven emotionalen Erfahrungen in Kontakt bringen.

Primäre Konstituenten von Persönlichkeits-
störungen
Diesem Ansatz zufolge frühkindliche mal-
adaptive Schemata (FMS), definiert als in ei-
nem signifikanten Maße dysfunktionale Le-
bensthemen, die sich aus Erinnerungen,
Gefühlen und Kognitionen zusammensetzen.
Sie sollen aus negativen Beziehungserfah-
rungen in der Kindheit erwachsen sein und
hindern den Patienten aktuell an einer be-
friedigenden Lebens- und Beziehungsgestal-
tung.

Copingstrategien. Schemata können durch zahlreiche Strategien bewusst gemacht werden. In Überschneidung mit den psychoanalytischen Konzepten von Widerstand und Abwehrmechanismen werden 3-maladaptive Bewältigungsstrategien vorausgesetzt, die die FMS perpetuieren ( $\bullet$ Abb.1, - Tab. 1).

- Erdulden (Freeze): Wenn Patienten Schemata erdulden, akzeptieren sie sie als wahr und richten ihr Leben danach aus. Ein Patient mit NPS, von einer emotional kühlen und distanzierten Mutter erzogen, entwickelt das Schema „emotionale Entbehrung, und fühlt sich repetitiv zu Frauen hingezogen, die diesem Muster entsprechen. 
Menschliche Grundbedürfnisse nach Young

I. sichere Bindung an andere (beinhaltet Sicherheit, Stabilität, unkonditionale Akzeptanz, Versorgung)

II. Autonomie, Kompetenz, Identitätsgefühl

III. Setzen realistischer Grenzen

IV. Freiheit, Wünsche und Gefühle auszudrücken

V. Spontaneität und Spiel

- Vermeiden (Flight) bedeutet, alles zu unternehmen, damit das Schema nicht mehr aktiviert wird. Menschen mit NPS sind in der Kunst der Vermeidung außergewöhnlich aktiv (der Patient geht überhaupt keine persönlichen Bindungen mehr ein oder konsumiert Alkohol oder Drogen, um sein emotionales Vakuum nicht spüren zu müssen).

- Überkompensation (Fight): Menschen, die überkompensieren, verhalten sich so, als sei das Gegenteil ihres Schemas wahr. Wer depriviert aufwuchs, fordert nun ständige Aufmerksamkeit, Sonderbehandlung, Sonderregeln.

Dies stellt den üblichen narzisstischen Copingstil im interaktionellen Kontext dar.

Grundbedürfnisse. Aus klinischen Beobachtungen und unter Einbeziehung anderer Theorien postuliert Young 5 zentrale und universelle Grundbedürfnisse menschlicher Existenz, deren spezifische Verletzung zu spezifischen Schemata führt.

\section{Schemata und Schemadomänen bei NPS}

\section{Schemadomäne I}

Die Schemadomäne I ( $\bullet$ Tab.2) resultiert typischerweise aus einem missbräuchlichen, instabilen, entwertenden, kalten und unvorhersehbaren Erziehungsstil. Auch wenn die Patienten äußerlich unantastbar wirken, ist diese eine besonders schwer zu therapierende Domäne, weil sie mit erheblichen Defiziten einhergeht und bei der NPS überdurchschnittlich ausgeprägt ist.

Missbrauch/Misstrauen. Das Schema Missbrauch/ Misstrauen spielt in der Entwicklung einer NPS eine nicht zu unterschätzende Rolle. Viele Patienten wurden von ihren Erziehern aufgrund ihrer Talente, Intelligenz oder anderer Fähigkeiten benutzt, um deren defizitäres Selbstwertgefühl zu stabilisieren. Eine häufige Konstellation besteht darin, dass männliche Patienten mit NPS von ihren Müttern als Partnersubstitut verwandt wurden, was den Grundstein für ihr ambivalentes Gefühl Frauen gegenüber legt. Sie sind immer auf der Hut. Unter traumatisierenden Umständen kam es zu teilweise sadistisch gefärbter Gewalt gegen den Aufwachsenden. Dass dieses Engramm psychotherapeutisch nicht einfach zu modifizieren oder zu löschen ist, ist evident.

Narzisstische Patienten übertreiben das Ausmaß, in dem andere sie vermeintlich benutzen und zweifeln an der Authentizität des Gegenübers.

Durch ihr oft einschüchterndes Verhalten machen es Narzissten ihren Mitmenschen sehr schwer, mit ihnen ehrlich umzugehen. Dennoch ist dies der einzige Weg, ihnen zu helfen. Auch wenn Of-

Tab. 1 Typische Erscheinungsformen von Copingstrategien.

\begin{tabular}{|ll}
\hline Copingstil & Typische Erscheinungsformen \\
\hline Erdulden & ausgeprägte Compliance, Dependenz \\
\hline Vermeidung & Sucht \\
& sozialer Rückzug \\
& ständige Stimulation, Workaholismus \\
\hline Überkompensation & Aggressivität \\
& Manipulation, Ausbeutung \\
& Dominanz \\
& Perfektionismus \\
\hline
\end{tabular}

\begin{tabular}{|c|c|c|}
\hline $\begin{array}{l}\text { II: Beeinträchtigung von Au- } \\
\text { tonomie und Leistung }\end{array}$ & $\begin{array}{l}\text { Kompetenz, Autono- } \\
\text { mie, Identitätsgefühl }\end{array}$ & $\begin{array}{l}\text { 6. Abhängigkeit/Inkompetenz } \\
\text { 7. Anfälligkeit für Schädigungen und Krankheit } \\
\text { 8. Verstrickung/unterentwickeltes Selbst } \\
\text { 9. Versagen }\end{array}$ \\
\hline $\begin{array}{l}\text { III: Beeinträchtigung im } \\
\text { Umgang mit Begrenzungen }\end{array}$ & $\begin{array}{l}\text { realistische Grenzen } \\
\text { und Selbstkontrolle }\end{array}$ & $\begin{array}{l}\text { 10. Anspruchshaltung /Grandiosität } \\
\text { 11. unzureichende Selbstkontrolle / Disziplin }\end{array}$ \\
\hline IV: Fremdbezogenheit & $\begin{array}{l}\text { Freiheit im Ausdruck } \\
\text { von Bedürfnissen und } \\
\text { Gefühlen }\end{array}$ & $\begin{array}{l}\text { 12. Unterordnung } \\
\text { 13. Selbstaufopferung } \\
\text { 14. Streben nach Zustimmung und Anerkennung }\end{array}$ \\
\hline $\begin{array}{l}\text { V: Wachsamkeit und Ge- } \\
\text { hemmtheit }\end{array}$ & $\begin{array}{l}\text { Spontaneität und } \\
\text { Spiel }\end{array}$ & $\begin{array}{l}\text { 15. Negativität/Passivität } \\
\text { 16. emotionale Gehemmtheit } \\
\text { 17. überhöhte Standards/übertrieben kritische Haltung } \\
\text { 18. Bestrafen }\end{array}$ \\
\hline
\end{tabular}

Tab. 2 Übersicht über Schemadomänen, Grundbedürfnisse und Schemata nach Young [15].

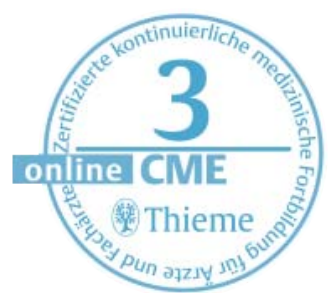


fenheit ambivalent erlebt wird, schätzen und brauchen sie die Patienten und respektieren erfahrungsgemäß nur ein Gegenüber, das den Mut aufweist, ihnen die Wahrheit mitzuteilen.

\section{Fallbeispiel Missbrauch/Misstrauen}

Ein 50-jähriger Informatiker meldete sich wegen einer schweren Erythrophobie bei einer Therapiestudie zur Behandlung sozialer Phobien an. Im SKID (Strukturiertes Klinisches Interview) wurde zusätzlich eine NPS diagnostiziert. Der Patient brach die Therapie trotz deutlicher Fortschritte nach wenigen Sitzungen mit dem Kommentar ab, er wolle nicht, dass die Behandler aus seiner Genesung einen Gewinn erzielen und „berühmt werden“ würden.

Emotionale Entbehrung. Bis auf wenige Ausnahmen ist nach diesem störungstheoretischen Modell die Entwicklung von NPS eng mit frühkindlichen Erfahrungen emotionaler Vernachlässigung verwoben. Das Schema erwächst beim Fehlen von unkonditionaler Liebe und beim Vorliegen eines emotional inhibierten, kühlen Interaktionsstils. Wenn es aktiviert wird, entstehen Emotionen von Einsamkeit und Unverstandensein.

Statt bedingungsloser Akzeptanz reagierten die Bezugspersonen mit Aufmerksamkeit und Anerkennung für Leistungen. Nach dieser Surrogaterfahrung hungert die unter NPS leidende Person ein Leben lang. Liebe kann nicht aufgenommen, verstanden oder erwidert werden, da sie fremd ist. Dennoch wird ihr Fehlen auf einer dem Bewusstsein nicht zugänglichen Ebene wahrgenommen. Auch führt dies zu einem extremen Potenzial an Neid. Wenn eine andere Person Lob oder Aufmerksamkeit erhält, entzündet sich bei den Betroffenen intensive Eifersucht. Zwischen Kindern aus NPS begünstigenden Familienumständen entbrennen oft heftige Kämpfe um die Aufmerksamkeit der Bezugspersonen, ein Phänomen, das Paulina F. Kernberg als „siblings intolerance“ beschrieb [16]

\footnotetext{
Fallbeispiel

Emotionale Entbehrung

Eine 40-jährige Lehrerin schildert, von ihren Eltern zeitlebens abgelehnt worden zu sein. Erst als während des Eislauftrainings ihre außergewöhnliche Begabung deutlich wurde, wandte sich ihre Mutter ihr zu. Im weiteren Verlauf muss sie unermüdlich trainieren, auf Kosten von Spaß und Entspannung. Vor den Auftritten hat sie extreme Angst, beherrscht sich aber, da die Sorge, die seltenen Momente der Aufmerksamkeit ihrer Mutter zu verlieren, dominiert. Als sie im Alter von 14 Jahren wegen eines Knorpelschadens ihre Karriere aufgeben muss, kehren die Eltern dazu zurück,
}

das Kind zu ignorieren. „Damit war ich für meine Eltern erledigt.“

Unzulänglichkeit/Scham. Das Schema „Unzulänglichkeit/Scham“ resultiert aus einer übermäßig kritischen, bloßstellenden und abwertenden Haltung der Bezugspersonen. Die Kritik wurde durch jedwedes Anzeichen von Mittelmaß verursacht. Häufig löste das Äußern normaler kindlicher Bedürfnisse Tadel aus. Daraus erwächst eine intensive Intoleranz für negative Rückmeldungen, hohe Empfindlichkeit und Evozieren von Beschämung, wenn die Personen bedürftig werden oder Fehler machen. Hierdurch erklärt sich vermutlich die erhöhte Suizidgefährdung. Beschämung wird meist jedoch virtuos verborgen, was insbesondere durch den Mechanismus der Externalisierung gelingt: eigene Fehler werden auf andere attribuiert.

Fallbeispiel

Scham

„Wenn ich mich einem Mann nähere und einen Korb bekomme, ist es für mich eine Katastrophe. Es fühlt sich an als müsste ich daran sterben.“

\section{Schemadomäne II}

Diese Domäne entsteht aus einem familiären Hintergrund, der die Entwicklung von Autonomie und Selbstbewusstsein durch Unterminierung oder Überprotektivität hemmt ( $\bullet$ Tab.2). Die meisten Personen mit NPS sind jedoch hyperautonom und, bedingt durch die Leistungsbezogenheit ihrer Umgebung, in Performanceangelegenheiten selbstsicher. Sie bitten selten um Hilfe und kommen in der Regel nicht auf angebotene Unterstützung in Krisenzeiten zurück. In Ausnahmefällen kann eine Unterform narzisstischer Ansprüchlichkeit im Sinne eines „dependent entitlement“ beobachtet werden [14].

\section{Schemadomäne III}

Dem emotionalen Vakuum, in dem die Patienten aufwuchsen, steht das Verwöhntwerden in materieller Hinsicht und die fehlende Grenzsetzung gegenüber ( $\bullet$ Tab. 2). Viele Familien aus diesem Formenkreis nehmen sich als „etwas Besseres“ wahr und lehren ihre Nachkommen, dass die Regeln, die für alle Welt Gültigkeit besitzen, für sie nicht zutreffend sind. Nicht selten werden einzelne Aspekte ihrer Talente überbewertet.

Anspruchshaltung/Grandiosität. Die Patienten sind der Überzeugung, dass sie tun und lassen können, was ihnen in den Sinn kommt und bekommen, was sie haben wollen, und zwar sofort. Sie stellen die Regeln auf, und brechen sie, wenn sie sich für sie als nicht zuträglich erweisen. 
Unzureichende Selbstkontrolle/Disziplin

Die Patienten tolerieren Langeweile und Routinetätigkeiten nur widerwillig und vermeiden neue Aufgaben, die ihnen nicht rasch gelingen.

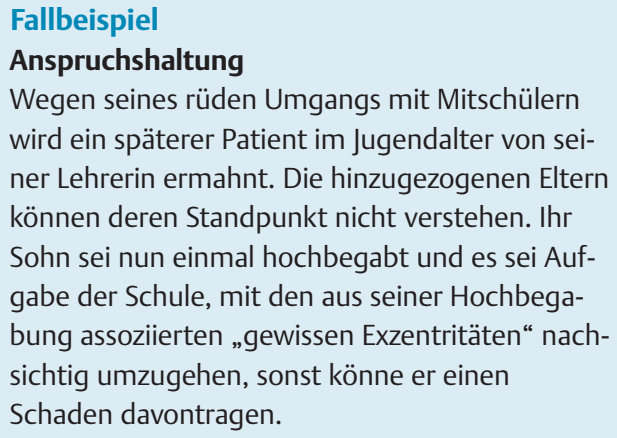

\section{Schemadomäne IV}

Die 4. Domäne ( $\diamond$ Tab. 2) entwickelt sich vor dem Hintergrund eines Klimas konditionaler Akzeptanz. So, wie der Heranwachsende ist, ist er nicht akzeptiert und muss wesentliche Aspekte seiner Persönlichkeit unterdrücken, um gesehen zu werden. Der spätere Narzisst hat so zu sein, zu denken und $\mathrm{zu}$ wünschen, wie es seine Umgebung für richtig erachtet. Viele Patienten sind sich ihrer eigenen Vorlieben gar nicht bewusst.

Unterordnung. Menschen mit NPS ordnen sich als autoritär oder machtvoller wahrgenommenen Gegenübern oft grollend unter, und äußern ihren angestauten Ärger nur indirekt. Andererseits wird Unterordnung in anderen Kontexten häufig überkompensiert: NP reagieren höchst allergisch auf Kontrolle und möchten grundsätzlich die Dinge in der Hand behalten.

Streben nach Zustimmung und Anerkennung. Das Schema ist fast durchgängig vorhanden. Hierbei wird der eigene Selbstwert von äußeren Bedingungen abhängig gemacht. Dem Wert von Geld, Status, Karriere und Äußerlichkeiten wird übertriebene Bedeutung beigemessen auf Kosten von genuiner Freude und Befriedigung.

\section{Schemadomäne V}

Schemadomäne V ( $\bullet$ Tab.2) entwickelt sich vor dem Hintergrund eines verbissenen, leistungsbezogenen, rigiden Umfeldes.

Überhöhte Standards. Personen mit NPS haben besonders mit extrem hohen Standards zu kämpfen. Sie streben nach Perfektionismus in allen Lebensbereichen. Mittelmaß ist phobisch besetzt, weckt es doch Erinnerungen an weit zurückliegende Zurücksetzungen und Beschämungen.

Im ständigen Hungern nach Anerkennung, und immer unter Druck, ihren Wert unter Beweis zu stellen erbringen die Patienten Leistung, meist auf hohem Niveau. An der eigentlichen Tätigkeit haben sie keine Freude.
Fallbeispiel

Überhöhte Standards

Motto einer Familie von Spitzenskiläufern: „Lieber tot als Dritter.“

\section{Einsatz des Moduskonzeptes}

$\nabla$

Menschen mit tiefergreifenden Persönlichkeitsstörungen weisen häufig extrem viele frühkindliche maladaptive Schemata (FMS) auf, sodass es uneffektiv, verwirrend und belastend erscheint, alle einzeln zu beleuchten. Therapeutische Techniken, die für ein FMS hilfreich sind, sind für ein anderes kontraindiziert: so muss Anspruchshaltung Grenzsetzung zur Folge haben, während diese für die Lebensthemen Unterordnung und emotionale Entbehrung nicht angewandt werden sollte. Hier wäre ein sorgfältiges Fokussieren auf die Wünsche und Bedürfnisse des Klienten von grundlegender Bedeutung. Darüber hinaus weisen Menschen mit NPS extrem rigide Copingstrategien von Vermeidung einerseits und Überkompensation andererseits auf, die es regelmäßig verhindern, Zugang zu den zugrunde liegenden FMS zu erhalten. Hieraus wird deutlich, wie die Entwicklung des Moduskonzeptes begriffen werden kann.

Ein Modus ist als ein zu einem bestimmten Zeitpunkt vorherrschender Affektzustand oder Persönlichkeitsanteil definiert, der Denken, Fühlen und Handeln bestimmt. Er umfasst die Gesamtheit der Schematrigger, Schemata und Bewältigungsstrategien, die aktuell in einer Person dominieren.

Als Analogie kann das „State versus-Trait“-Konzept herangezogen werden. Demnach ist ein „Trait“ eine Persönlichkeitseigenschaft, die eine zeitstabile Variable darstellt und hier in dem Begriff „Schema“ aufgeht, wohingegen ein „State“ einen Zustand beschreibt, der variiert und dem Ausdruck „Modus“ entsprechen würde.

Es wird davon ausgegangen, dass Modi bei jedem Menschen zu beobachten sind und in einem Spektrum von funktional (=gesund) bis zu dysfunktional (=pathologisch) rangieren. Ein psychologisch gesundes Individuum kann die verschiedenen Anteile seines Selbst integrieren und ein Identitätsbewusstsein aufrechterhalten. Je schwerer gestört ein Patient ist, desto unintegrierter stehen die Modi nebeneinander. Unterschieden werden kindliche Modi, elterliche Modi im Sinne einer Internalisierung von Erziehungsgrundsätzen, kompensatorische Modi in Anlehnung an die oben erwähnten Copingstile und der Modus des gesunden Erwachsenen. Für die NPS werden der verletzbare kindliche Modus, der den

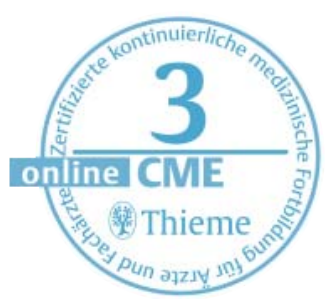




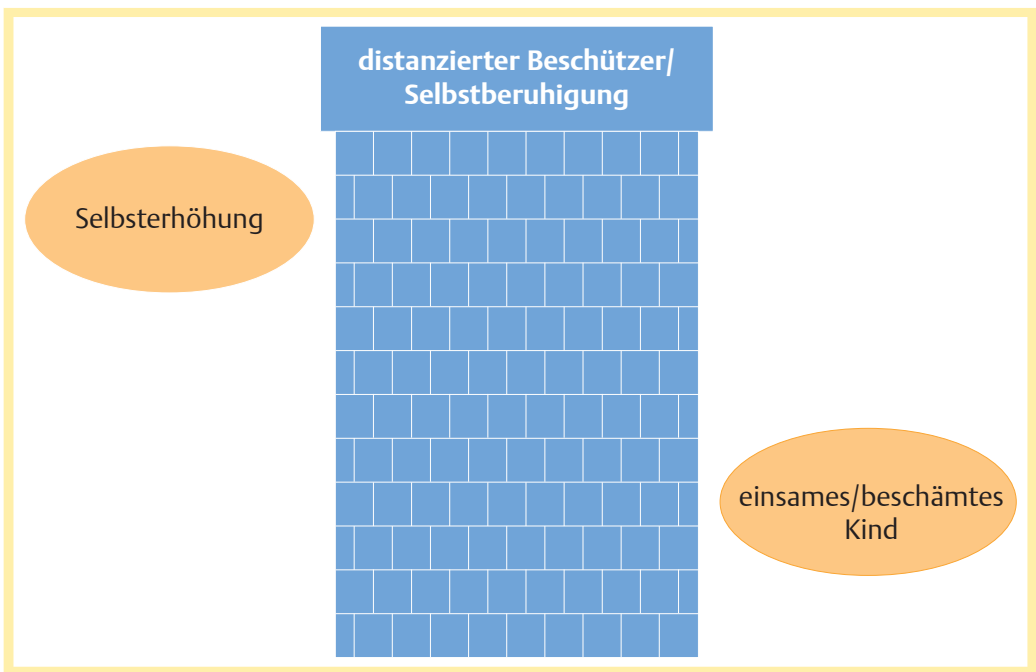

Abb.2 Narzisstische Modi.

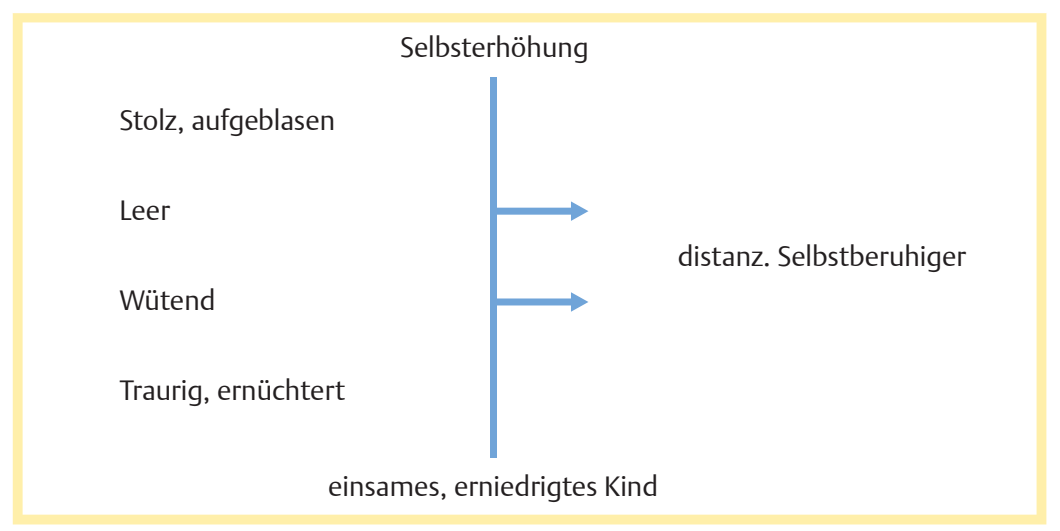

Abb. 3 Dynamik der Schemamodi in der NPS.

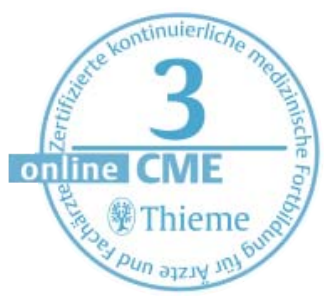

Kern der Psychopathologie beherbergt, ein überkompensierender sowie ein vermeidender Modus postuliert ( $\bullet$ Abb. 2).

Durch das Moduskonzept wird es möglich, die therapeutischen Interventionen und die Haltung dem jeweilig aktuell dominierenden Zustand des Patienten anzupassen. Statt den Patienten in seiner Gesamtheit anzusprechen, werden verschiedene Seiten in ihm adressiert. Dieses Vorgehen führt zu einer Entstigmatisierung und fördert die Zusammenarbeit.

- Abb. 3 nach A. Arntz (persönliche Mitteilung) zeigt die Dynamik der Schemamodi.

\section{Modus der narzisstischen Selbsterhöhung}

Dies ist der Modus, dessen sich Personen mit NPS üblicherweise im Kontakt mit anderen bedienen, er entspricht dem Copingstil der Überkompensation. Er ist in hohem Maße ich-synton und wird aus der Eigenperspektive selten als störend erlebt. „Erfolgreiche“ Narzissten verbringen die meiste Zeit in diesem Zustand. Die unterliegenden FMS sind Ansprüchlichkeit, Streben nach Anerkennung, Misstrauen, Unterordnung und überhöhte Standards. Wenn der Modus aktiviert wird, verhalten sich die Patienten überheblich, arrogant, ansprüchlich, kritisch, eitel, ichbezogen oder prahlen mit ihren Errungenschaften oder Talenten. Sie ergehen sich in Phantasien von Macht und Brillanz. Sie sind manipulativ und beuten ihre Umgebung aus. Sie kreieren ihre eigene „Wahrheit“, um sich überlegen, perfekt und makellos zu fühlen. Da die eingeschüchterte Umgebung sich meist nicht traut, ihre Lügen zu korrigieren, glauben sie sie irgendwann selbst. Empathie wird nur dazu benutzt, die Schwächen der anderen zu identifizieren und zu benennen. Ansonsten wird sie abgestellt, was dazu führt, dass sich die Patienten wenig Gedanken machen, was in den Köpfen ihrer Mitmenschen vor sich geht und erstaunt sind, wenn sie plötzlich auf Ablehnung stoßen. Die Umwelt muss kontrolliert werden, da sie als bedrohlich und manipulativ eingeschätzt wird. Ein Bedürfnis zu haben oder sich auf andere stützen können, ist inakzeptabel. Dieser Modus folgt dem Motto „Angriff ist die beste Verteidigung“. Kommunikation wird als Zweikampf erlebt, bei dem es nur Gewinner oder Verlierer geben kann. Das zugrunde liegende Motiv dieses Zustandes besteht darin, vor Gefühlen von Unterlegenheit, Scham und Isolation zu schützen und Anerkennung und Lob zu erhalten. Tragischerweise konterkariert der Modus das Motiv: die Bezugspersonen ziehen sich früher oder später entnervt und ernüchtert zurück. Der Patient mit NPS erreicht den Zustand, den er eigentlich um jeden Preis vermeiden wollte: Einsamkeit und Unverständnis.

\section{Therapieziele}

- Die Patienten sollen verstehen, wann und warum sie diese Seite entwickelt haben.

- Sie sollen die Vorteile dieses Modus verstehen und sich seiner Nachteile bewusst werden.

- Der Modus soll im Verlauf der Therapie in den Hintergrund treten, die Sucht nach Anerkennung, Kontrolle und Erfolg soll zugunsten der Etablierung reziproker Beziehungen in den Hintergrund treten.

Dies ist schwierig zu bewerkstelligen. Üblicherweise kommen Narzissten in Behandlung, wenn Fehlschläge ihre Kompensationsstrategien haben versagen lassen. Da sie jedoch eine starke Tendenz zur Externalisierung aufweisen, wünschen sie sich Therapeuten, die sie in ihrer Innenperspektive im Kampf gegen die Umwelt unterstützen und sich mit ihnen gegen dieselbe verbünden, um die Misserfolge ungeschehen zu machen. Sie sind von der Vorstellung geleitet, dass die Kompensationsstrategien wieder etabliert werden und selten zu einer kollaborativen Exploration des Störungscharakters ihres interaktionellen Stils bereit. 
Fallbeispiel

\section{Externalisierung}

Wegen seiner habituellen Affären wird ein Patient mit NPS von seiner Frau verlassen. Er äußert sich folgendermaßen: „Osama bin Laden ist letztlich schuld am Scheitern meiner Ehe. Meine Frau war nach den Terroranschlägen einfach wie verwandelt."

Auch haben die Patienten bislang alles unternommen, sich nicht vulnerabel zu zeigen, d.h. alleine die Tatsache, Hilfe nötig zu haben, ist exzessiv beschämend.

Darin gründet der starke Wunsch, Behandlung und Behandler abzuwerten.

\section{Fallbeispiel \\ Abwertung und Kontrolle}

Der 50-jährige Informatiker aus dem Fallbeispiel von S. 60 bringt seinem Behandler in der 3. Therapiestunde das von ihm vollständig durchkorrigierte und mit Kommentaren versehene Merkblatt zur Genese von sozialen Phobien mit. Dieses sei „defizitär“. Seinen vorzeitigen Therapieabbruch begründet er mit den Worten, er werde nun selbst einen Therapieansatz zur Behandlung von Erythrophobien ausarbeiten und veröffentlichen.

\section{Hilfestellungen im Umgang mit der Selbsterhöhung}

Etablierung von Gegenseitigkeit. Es ist von grundlegender Bedeutung, auf respektvollem Umgang zu bestehen. Patienten mit NPS achten ausschließlich ein Gegenüber, dass sich von ihnen nicht abwerten lässt. Dementsprechend sollte man deutlich machen, dass die interaktionellen Regeln, die man als Therapeut selbstverständlich zu befolgen bereit ist (angemessener Tonfall, keine Beleidigungen oder Bedrohungen), auch vom Patienten erwartet werden. Im Umgang mit der Selbsterhöhung wählt der Therapeut einen konfrontativeren und weniger zugewandten Stil. Es ist oft sinnvoll, den narzisstischen Patienten die Konsequenzen seines Verhaltens spüren zu lassen.

Die günstigste Haltung im Umgang mit dem Modus der Selbsterhöhung ist die Annahme, dass sich der narzisstische Patient dieser Seite in aller Regel nicht bedient, um böswillig zu verletzen, sondern um sich zu schützen. Dadurch wird es leichter, eine empathische Grundhaltung beizubehalten.
Praktisches Vorgehen

Selbsterhöhung

„Wie würden Sie sich fühlen, wenn ich Ihnen das so vermitteln würde? Gekränkt? Ja, sehen Sie, so ist es mir auch gegangen."

Manchmal genügt es, auf eine abwertende oder unverschämte Bemerkung einen Moment des Schweigens folgen zu lassen, damit der Patient der Wucht seiner Worte lauschen kann. Wenn man scharf angegriffen wird, ist es entscheidend, sich nicht zum Gegenangriff verleiten zu lassen und stattdessen auf die Metaebene zu wechseln. „Warum möchten Sie so mit mir umgehen?“ Diskutieren über Inhaltliches ist in aller Regel in diesen Fällen ein therapeutischer Fehler.

Von eminenter Bedeutung ist auch, zu einem Modell für das Eingestehen eigener Schwächen zu werden. Und sich bei Fehlern zu entschuldigen.

Darüber hinaus verwendet der Therapeut die Modussprache. Er adressiert den Patienten nicht in seiner Gesamtheit, sondern spricht „die Seite in Ihnen“ oder „der Teil von Ihnen“ an. Hierdurch kommt es zu einer Entpathologisierung und Verminderung des Widerstands.

\section{Praktisches Vorgehen \\ Modussprache \\ „Was ist das für eine Seite in Ihnen, die sich \\ Menschen in Form eines Angriffs nähert, um sich selbst zu schützen?“ \\ „Ich würde gerne diese Seite an Ihnen besser kennenlernen, die das Bedürfnis hat, mich herabzusetzen."}

\section{Interventionen}

Empathische Konfrontation. Zentrale Technik im Umgang mit der Seite des Selbsterhöhens ist die Strategie der empathischen Konfrontation. Im Hier und Jetzt der therapeutischen Beziehung werden problematische Verhaltensmuster aufgezeigt.

- Der Therapeut äußert Verständnis dafür, warum der Patient die Welt sieht, wie er es tut und konfrontiert den Modus.

- Er zeigt sich einfühlsam bezüglich der Gründe, warum der Patient den Modus entwickelt hat und wie schwierig Veränderung ist.

- Gleichzeitig betont er die Notwendigkeit des Wandels.

- Er bemüht sich hierbei um optimale Balance.

Praktisches Vorgehen

\section{Empathische Konfrontation}

„Ich weiß, dass das schwierig für Sie ist, einmal warten zu müssen, da niemand Ihnen beigebracht hat, dass die Regeln, die für alle gelten, auch für Sie gemacht worden sind. Und Ihnen wurde erklärt, dass Ihnen besondere Privilegien

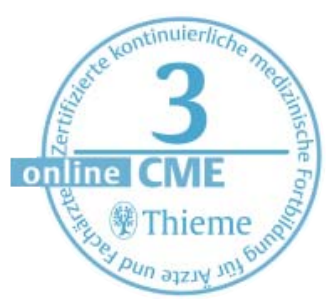


zustehen. Also ist es nicht Ihre Schuld. Ich bin psychologisch ausgebildet und kann Ihre Sichtweise nachvollziehen. Aber alle anderen kennen diesen Hintergrund nicht und werden deshalb von Ihrem Verhalten verärgert sein."

CAVE: Die Technik der empathischen Konfrontation funktioniert nur, wenn Therapeuten genuines Mitgefühl für den Patienten aufbringen können.

Kognitive Techniken. Es werden Pro- und Kontralisten über die Vor- und Nachteile des Bewältigungsmodus erörtert. Zur Identifizierung kognitiver Verzerrungen werden Gedankentagebücher geführt, in denen Patienten in schwierigen Situationen ihre automatischen Gedanken notieren und korrigieren. Insbesondere das ausgeprägte Schwarz-Weiß-Denken wird hinterfragt.

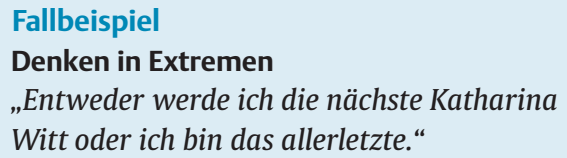

Modusdialoge. Der Patient wird aufgefordert, im Rahmen eines Stuhldialogs zunächst ganz zu der Seite zu werden, die dem Modus des Selbsterhöhens entspricht. Auf einem anderen Stuhl soll die Seite Platz nehmen, die dem einsamen/beschämten Kindmodus entspricht.

\section{Praktisches Vorgehen \\ Stuhldialog}

„Werden Sie einmal ganz zu der Seite in Ihnen, die denkt, eine Lebensberechtigung hat nur, wer ein Eislaufstar wird. Lassen Sie mich hören, was diese Seite vorzubringen hat. Und lassen Sie mich hören, warum sie meint, dass die 14-jährige Julia mit ihrem kaputten Knöchel so unglaublich verachtenswert ist.“

„Sie haben einen Anteil, der unglaublich streng zu sich ist und sich sagt, dass Sie den ganzen Tag hart trainieren müssen. Gibt es einen Teil, der diese Stimme hört? Und wie geht es dem damit?“

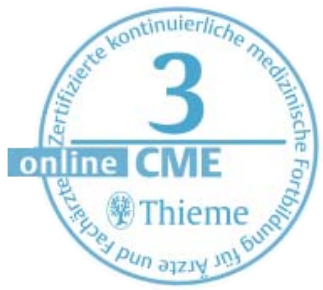

\section{Behaviorale Techniken.}

- Rollenspiele zum Einüben funktionaleren Verhaltens, Lernen am Modell.

- Videofeedback und Videoanalysen zur Vermittlung von Kompetenzerwerb.

- Verhaltensexperimente (Zeit mit sich alleine verbringen, explizites Fokussieren auf das Gegenüber, Einlegen von Sprechpausen).

- Führen von Tagebüchern über auslösende Variablen, dysfunktionale Selbstinstruktionen, Reaktionen der Umgebung.
Modus des einsamen/beschämten Kindes Wenn für die NPS die Quellen von Anerkennung oder Beachtung versiegen, wenn sie über einen längeren Zeitraum alleine sind, wenn sie zurückgewiesen, verlassen oder kritisiert werden oder wenn sie keinen Erfolg haben, wird der Modus des einsamen Kindes getriggert. In diesem Zustand erleben die Patienten die mit den FMS Emotionale Entbehrung, Unzulänglichkeit/Scham und Unterordnung verbundenen Gefühle von Einsamkeit, Scham und Abwertung. Sie fühlen sich als Mittelmaß, ungeliebt, ignoriert, vorgeführt und leer und werden depressiv, wenn es nicht durch Aktivierung ihrer Kompensationsstrategien gelingt, den Zustand zu beenden.

Der Modus des einsamen/beschämten Kindes ist derjenige, den die Patienten vermeiden und der Therapeut erreichen möchte, da hier die fruchtbarste Arbeit stattfinden kann.

\section{Therapieziele}

- Das Konzept des einsamen Kindes muss eingeführt und ins Bewusstsein des Patienten gerückt werden.

- Die damit assoziierten Gefühle wie Einsamkeit und Scham müssen zu Tage treten dürfen, um ausreichend Motivation für Veränderung zu generieren.

- In diesem Modus soll der Patient erleben, sich unterstützt, akzeptiert und angenommen zu fühlen.

- Psychoedukation soll vermitteln, welche Bedürfnisse Kinder naturgemäß haben und inwieweit sie für den Patienten nicht erfüllt wurden, um Mitleid statt Verachtung für die vulnerablen Anteile zu generieren [17].

Die wenigsten Patienten wissen um die Existenz dieser Seite. Wenn sie dennoch einen Zugang zu diesem Persönlichkeitsanteil erlangen, ist er hochgradig aversiv besetzt und von Verachtung geprägt. Es wird alles unternommen, um sich nicht vulnerabel zu machen.

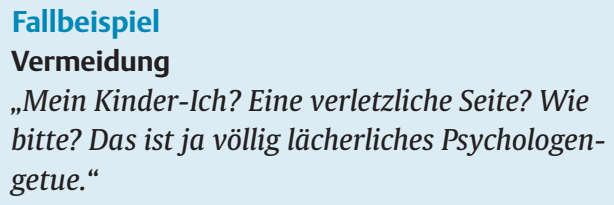

Hilfestellungen für den verletzlichen Modus Das Gehirn ins Spiel bringen. Eine Möglichkeit des Umgangs mit Abwehr ist es, den Patienten ein biologisches Erklärungsmodell zu bieten [18]. 
Praktisches Vorgehen

Biologisches Erklärungsmodell

„Dann nennen Sie diese Seite eben die Seite ihrer unangenehmen Erinnerungen. Ihr implizites Gedächtnis hat Erinnerungen an belastende Erlebnisse während des Aufwachsens gespeichert, jedoch sind diese und die dazugehörigen Gefühle so tief eingebettet, dass Ihnen das nicht bewusst ist. So funktioniert das Gehirn nun mal. Sie erinnern sozusagen, ohne sich daran zu erinnern. Wir wissen das aus der neurobiologischen Grundlagenforschung. Und wenn dann ein aktuelles ähnliches Erlebnis passiert, kann Ihr Gehirn nicht zwischen damals und heute unterscheiden, sieht eine Bedrohung, die neuronalen Strukturen feuern, und es werden intensive Emotionen produziert. Ihr Hirn reagiert sozusagen auf ein Gespenst aus der Vergangenheit."

Fotoalbum. Ebenfalls günstig kann sich das gemeinsame Durchblättern alter Fotoalben auswirken. Häufig transportiert sich dadurch die Einsamkeit, die dem Patienten nicht bewusst ist. Auch hier kann der Widerstand konfrontiert werden. Der Therapeut deutet auf ein Bild und fragt den Patienten, ob er allen Ernstes die Existenz dieses Kindes in seinem Denken und Fühlen bestreiten möchte.

Imagination. Wird im Kontext der therapeutischen Beziehung ein FMS aktiviert, kann der Patient aufgefordert werden, die Augen zu schließen und sich eine Situation aus der Kindheit vorzustellen, die sich ähnlich anfühlte.

\section{Modus des distanzierten Beschützers| der distanzierenden Selbstberuhigung} Wenn der Modus des einsamen Kindes nicht mehr toleriert werden kann (oder, wenn sie alleine sind), wechseln die Patienten in den Modus der Selbstberuhigung. Er entspricht dem Konzept der Schemavermeidung und dient dazu, unangenehme Gefühle von Scham oder Einsamkeit abzustellen. Dabei werden Ablenkungsmanöver initiiert, die häufig suchtartigen Charakter annehmen: Arbeiten bis zur Erschöpfung, Essanfälle, Drogen- und Alkoholmissbrauch, übermäßige Beschäftigung mit dem Computer. Auch stimulierende Strategien können verwandt werden, um negative Gefühle unter Kontrolle zu halten: riskantes Fahrverhalten, Spekulieren an der Börse, Kleptomanie. Menschen mit NPS werden sehr weit gehen, um nicht mit ihrer verletzlichen Seite konfrontiert werden zu müssen. In Extremfällen weisen sie dissoziatives Verhalten auf und vergessen unangenehme Ereignisse. Auch im Kontakt zu anderen bleiben die Patienten distanziert. Das führt dazu, dass sie sich geschützt fühlen und erleichtert es ihnen, ihre Umgebung als Objekte wahrnehmen zu können. Kontakte bleiben unbelebt, wodurch die Fähigkeit, die Umwelt ohne ausgeprägtere Skrupel benutzen zu können, geför- dert wird. Ungünstigerweise intensiviert dieser Modus jedoch das Erleben von Einsamkeit.

\section{Fallbeispiel/Praktisches Vorgehen Imagination}

Die 40-jährige Lehrerin aus dem Fallbeispiel S. 60 weint, als sie befürchtet, wegen nicht gemachter Hausaufgaben die Therapeutin verärgert zu haben. In der Imagination sieht sie sich nach einem Eislaufturnier alleine im Bus sitzen. Die Eltern haben sich geweigert, neben ihr Platz zu nehmen.

Sie ist nur zweite geworden. Als sie weint, wird sie nachdrücklich vor allen Fahrgästen kritisiert. Sie schwört sich, in Zukunft immer die Beste zu sein. Und niemals jemandem anderen wieder eine Schwäche zu offenbaren.

In diesem Fall betritt der Therapeut das Bild, verteidigt das Kind vor dysfunktionalen Botschaften der anderen Anwesenden, klärt Eltern oder andere Bezugspersonen über die universellen Rechte und Bedürfnisse von Kindern auf. Er sucht den Dialog zu dem Kind, validiert dessen Gefühle und Reaktionen und lässt sich seine Ängste und Wünsche schildern. Auch kann im Bild das Kind visuell verdoppelt werden: in die Seite, die leidet (das beschämte Kind) und die Seite, die nie wieder zweite werden wird (die Seite der Selbsterhöhung). Mit beiden Teilen des Selbst kann der Therapeut in einen Dialog treten.

\section{Therapieziele}

Auch dieser Modus soll im Verlauf der Behandlung geschwächt werden zugunsten des Eingehens naher, vertrauensvoller Beziehungen und des Abbaus von Interaktionsstörungen.

\section{Hilfestellungen für die distanzierende \\ Selbstberuhigung}

Um die Therapiemotivation aufrechtzuhalten, ist es sinnvoll, immer wieder auf die negativen Konsequenzen des Verhaltens einzugehen.

Modusdialoge. Auch hier empfiehlt sich ein Stuhldialog mit dem Anteil, der sich schützt. Es ist günstig, wenn die Patienten selbst Namen für ihre jeweiligen Modi wählen. So benannte die bereits erwähnte Eisläuferin die Seite an sich, die keine Gefühle mehr spüren oder zeigen wollte als „die toughe Julia“.
Praktisches Vorgehen
Stuhldialog
„Ich möchte Sie bitten, einmal ganz zu der Seite in Ihnen zu werden, die sich so hart gemacht hat. Und stellen Sie sich vor, auf dem Stuhl neben Ihnen nimmt die Seite Platz, die 7 Jahre alt ist und sich mehr Kontakt und Nähe wünscht." Der Dialog könnte folgendermaßen lauten: „Seit wann sind Sie schon im Leben von Frau M. exis- 
tent? Wann wurden Sie zum ersten Mal aktiv? Mit welchem Ziel? Wobei helfen Sie ihr? Und wie genau sieht das aus? Was sind die Vorteile Ihrer zeitweiligen Anwesenheit? Gibt es auch Nachteile?“ Nach der Übung wechselt die Patientin auf den Stuhl, der die Seite des Kindes repräsentiert und kann Trauer über die Einsamkeit und Isolation, die der distanzierende Modus mit sich bringt, äußern.

Die verschiedenen Anteile der Patientin werden quasi zu Personen, die miteinander in Verhandlung treten. So wird es möglich, statt darüber zu sprechen, warum eine Person intime Kontakte vermeidet, mit dem Teil in ihr zu sprechen, die dies als so wichtig erachtet.

Es ist bedeutsam, die Funktion dieses Modus verstanden und seine Funktion als Überlebenshilfe in der Vergangenheit validiert zu haben, bevor man auf die Schwierigkeiten, die aus seiner Existenz resultieren, eingeht. Das Modusverhalten kann umso eher in den Hintergrund treten, je mehr sich dieser Teil des Patienten verstanden fühlt.

Audiomemos. Patienten mit NPS schätzen es unserer Erfahrung nach, Audiomemos mit Instruktionen des Therapeuten für schwierige Situationen zu erhalten [19].

„Ich weiß, dass Sie ein schwieriges Wochenende vor sich haben, weil Sie alleine sein werden und dass das Bemühen nach Ablenkung durch Stehlen sehr intensiv werden wird. Aber wir arbeiten gemeinsam daran, dass Ihr Leben einen glücklicheren Weg nehmen wird. Versuchen Sie, mit der Seite an Ihnen, die sich zurückgelassen und einsam fühlt, Kontakt aufzunehmen. Und nehmen Sie sich einen Moment Zeit, diesen Teil zu trösten/ zu beruhigen/ zu stärken.“

\section{Fazit und Ausblick}

Narzissmus in seiner ausgeprägten Form ist eine sehr belastende Störung, die das Selbstbild eines Menschen, sein Gefühlsleben, seine Einstellungen und sein Verhalten zutiefst beeinflussen und für viel Leid sorgen kann. Veränderte gesellschaftliche Bedingungen fördern die Dialektik von Grenzenlosigkeit bei parallel bestehendem zunehmenden Verlust von kindlicher Geborgenheit. Damit bereiten sie den Boden für die Entwicklung von vermeintlich übersteigertem Selbstbewusstsein bei innerlicher Orientierungslosigkeit.

Kompensatorische Strategien zur Selbstregulation wie Fokussieren auf Macht, Status, Äußerlichkeiten und extreme Abwertungen anderer werden nicht zuletzt durch die Omnipräsenz und Ausgestaltung der medialen Landschaft verstärkt und gesellschaftsfähig. Die Inzidenz narzisstischer Störungen nimmt zu. Die Folgen für unsere Gesellschaft sind nicht absehbar.
Kernaussagen

- Narzisstische Persönlichkeitsstörungen gelten gemeinhin als nicht einfach zu therapieren.

- Häufiger als bei anderen Störungen besteht die Gefahr, dass die Patienten für sich, aber auch für ihre Umgebung, durch suizidales oder fremdaggressives Verhalten zu einer Gefahrenquelle werden.

- Die ich-syntonen, rigiden Vermeidungs- und Überkompensationsstrategien erschweren den Blick auf die verletzliche Seite der Patienten sehr häufig.

- Es ist ein hohes Maß an therapeutischer Balance und Flexibilität zwischen Empathie, Aufrichtigkeit und Warmherzigkeit und einem konfrontativen und Grenzen setzenden Verhalten vonseiten des Therapeuten notwendig, um für diese Patienten hilfreich zu sein.

- Die Schematherapie bietet einen integrativen Ansatz, der entwicklungsbedingtes Fallverständnis mit kognitiven, behavioralen und emotionsfokussierenden therapeutischen Strategien verbindet. Und damit hoffentlich dazu beitragen kann, den Leidensdruck der Patienten und die Belastungen, die sie ihrer Umwelt zufügen, zu minimieren.

Interessenkonflikt: Die Autoren geben an, dass kein Interessenkonflikt besteht.

\section{Literatur}

1 Fiedler P. Persönlichkeitsstörungen. Weinheim: Beltz; 2007

2 Smith BenjaminL. Die interpersonelle Diagnose und Therapie der Persönlichkeitsstörungen. München: CIP Medien; 2001

3 Bernstein DP, Arntz A, de Vos M. Schema Focused Therapy in Forensic Settings: Theoretical Model and Recommendations for Best Clinical Practice. International Journal of Mental Health 2007, (6): 169-183

4 Twenge JM, Campbell WK. The Narcissism Epidemic. Living in the Age of Entitlement. New York: Free Press; 2009

5 Stinton FS, Dawson DA, Goldstein RB. Prevalence, Correlates, Disability, and Comorbidity of DSMIV Narcissistic Personality Disorder: Results from the Wave 2 National Epidemiologic Survey on Alcohol and Related Conditions. J Clin Psychiatry 2008, (69): 1033-1045

6 Fiedler P. Integrative Psychotherapie bei Persönlichkeitsstörungen. Göttingen: Hogrefe; 2000

7 Cooper AM. Further Developments in the Clinical Diagnosis of Narcissistic Personality Disorder. In: Ronningstam EF, Hrsg. Disorders of Narcissism. Washington: American Psychiatric Press; 1998

8 Millon P. Modern psychpathology: A biosocial approach to maladaptive learning and functioning. Philadelphia: W. B. Saunder; 1969

9 Millon P. DSM Narcissistic Personality Disorder: Historical Reflections and Future Directions. In: Ronningstam EF Hrsg. Disorders of Narcissism. Washington: American Psychiatric Press; 1998

10 Millon P. Disorders of Personality: DSM IV and beyond. New York: Wiley; 1996 
11 Leary T. Interpersonal Diagnosis of Personality. New York: Roland; 1957

12 Sachse R. Histrionische und narzisstische Persönlichkeitsstörungen. Göttingen: Hogrefe; 2002

13 Beck AT, Freeman A. Kognitive Therapie der Persönlichkeitsstörungen. Weinheim: Beltz; 1999

14 Young J, Klosko JS, Weishaar ME. Schematherapie. Ein praxisorientiertes Handbuch. Paderborn: Junfermann; 2005

15 Jacob G, Bernstein D, Lieb K et al. Schematherapie mit dem Moduskonzept bei Persönlichkeitsstörungen. Psychiatr Psychother up2date 2009; 3: 105-120

16 Kernberg PF. Developmental Aspects of Normal and Pathological Narcissism. In: Ronningstam EF, Hrsg.
Disorders of Narcissism. Washington: American Psychiatric Press; 1999

17 Jacob GA, Bernstein DP, Lieb K et al. Schematherapie bei Borderlinepersönlichkeitsstörung. In: Sachsse U, Herpertz SC, Kernberg OF et al., Hrsg. Handbuch Borderlinestörungen. Stuttgart: Schattauer in Druck

18 Behary W. Disarming the Narcissist: Surviving and Striving with the Self Absorbed. Oakland: New Harbinger Publications; 2008

19 Dieckmann E. Die narzistische Persönlichkeit mit Schematherapie behandeln. Stuttgart: Klett-Cotta; 2011 


\section{CME-Fragen Schematherapie: Ein Ansatz zur Behandlung narzisstischer Persönlichkeitsstörungen}

1 Welches ist kein DSM-IV-Kriterium der narzisstischen Persönlichkeitsstörung?

A Mangel an Empathie

B zwischenmenschlich ausbeuterisches Verhalten

C Phantasien von Macht und Erfolg

D chronische Gefühle von Einsamkeit und Leere

E Arroganz

Welches Schema ist bei der NPS nicht regelhaft anzutreffen?

Anspruchshaltung/ Grandiosität

Streben nach Zustimmung und Anerkennung

Unzulänglichkeit /Scham

Selbstaufopferung

überhöhte Standards/übertrieben kritische Haltung

Wie wird mit dem Modus der Selbsterhöhung in der Schematherapie umgegangen?

Es wird vermieden, ihn zu konzeptualisieren.

Er wird ignoriert.

Er wird empathisch konfrontiert.

Er wird unterstützt und bestärkt.

Er darf auf keinen Fall angegangen werden.

Durch welche Moduskombination ist die narzisstische Persönlichkeitsstörung gekennzeichnet? einsames Kind/distanzierte Selbstberuhigung/Selbsterhöhung

B undiszipliniertes Kind/strafender Elternteil/gesunder Erwachsener

C glückliches Kind/übermäßige Kontrolle/bereitwilliges Erdulden

D verlassenes Kind/fordernder Elternteil/distanzierter Beschützer

E verlassenes Kind/Schikane und Angriff/ärgerliches Kind

Welche Antwort ist falsch?

A Die NPS geht mit einer erhöhten Suizidneigung einher.

B Misserfolge und Rückschläge können depressive Krisen auslösen.

c Hinter ihrer arroganten Fassade sind viele Patienten ausgesprochen schüchtern.

D NPS können aggressiv und bedrohlich reagieren.

E Die Störung ist unbehandelbar.

\section{6}

A frustriertes Grundbedürfnis

B Modus

C Copingstrategie

D unbewusster Trieb

E Schema

7

A

B

C

D

E Erlernen des kognitiven Behandlungsmodells

8 Welcher elterliche Erziehungsstil fördert nach Annahme der Schematherapie die Entwicklung von Narzissmus?

A Angemessene Grenzsetzung

B Gewährung von Autonomie

C Ermöglichen von Spontaneität und Spiel

D Konditionale Akzeptanz

E Unkonditionale Zuwendung

9 Welche Aussage ist für den Modus des distanzierten Beschützers zutreffend?

A Er ist selten vorhanden

B Er wird als Ausdruck gesunden Verhaltens konzeptualisiert

C Er dient dazu, negative Gefühle abzustellen

D Er soll im Verlauf der Behandlung verstärkt und ermutigt werden

E Er hilft dem Patienten, emotionale Nähe zu generieren

10

A Fokus auf die Therapiebeziehung im Sinne der „begrenzten elterlichen Fürsorge“

B Audiomemos

C Traumdeutung

D Stuhldialoge

E Imaginationsübungen

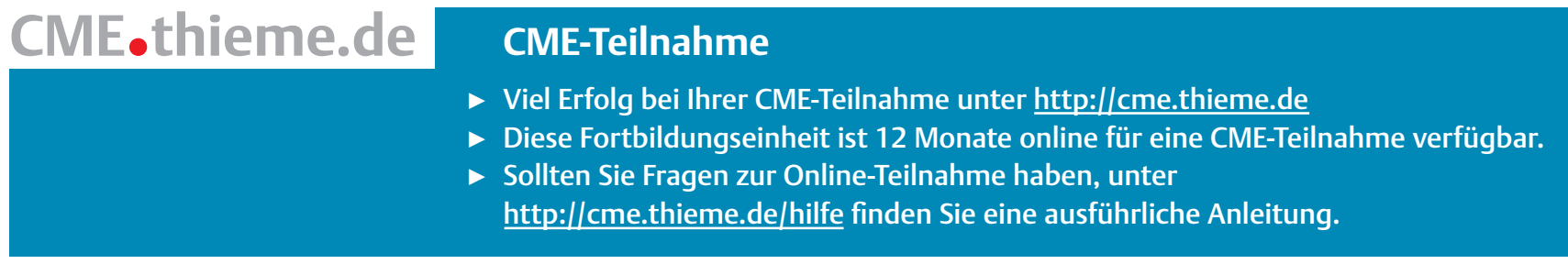

